\title{
The need for BT in ELBW
}

\author{
Raniyah E. D. Alharbi ${ }^{1}$, Zaki G. Alhothali ${ }^{2}$, Abdulrahman S. Altalhi ${ }^{2}$, Nemer K. Alghamdi ${ }^{2}$, \\ Naif M. Althobaiti ${ }^{2}$, Anas M. Alqadi ${ }^{2}$, Hassan A. H. Almalki ${ }^{2}$ \\ ${ }^{1}$ Pediatric intensivist, Saudi Arabia \\ ${ }^{2}$ Pediatric resident, Saudi Arabia
}

*Corresponding Author: Zaki G. Alhothali; Zakialhothli@hotmail.com

Received 27 April 2021;

Accepted 15 May 2021;

Published 25 May 2021

\begin{abstract}
Infants of extremely low birth weight usually receive multiple transfusions of red blood cells, typically in response to planned hemoprotein or haematocrit thresholds. Limited knowledge recommends that higher haemoglobin thresholds for red-cell transfusions could cut back the chance of psychological feature delay among extremely-low-birth-weight infants with anemia. Within the absence of higher indices, hemoprotein levels are imperfect however necessary guides to the necessity for transfusion. Chronic anaemia in premature infants could, if severe, cause apnea, poor neurodevelopmental outcomes or poor weight gain. On the opposite hand, red corpuscle transfusion could lead to transmission of infections, circulatory or pathology, or dysfunctional chemical element carriage and delivery. Hemoglobin is formed of four macromolecule molecules (globulin chains) that ar connected along. The traditional adult haemoglobin (abbreviated Hgb or $\mathrm{Hb}$ ) molecule contains 2 alpha-globulin chains and 2 beta-globulin chains. In fetuses and infants, beta chains aren't common and also the haemoglobin molecule is formed of 2 alpha chains and 2 gamma chains. Because the baby grows, the gamma chains are step by step replaced by beta chains, forming the adult haemoglobin structure. Each simple protein chain contains a vital iron-containing pigment compound termed pigment. Embedded among the pigment compound is associate degree iron atom that's important in transporting chemical element and carbonic acid gas in our blood. The iron contained in haemoglobin is additionally accountable for the red color of blood. Hemoglobin additionally plays a vital role in maintaining the form of the red blood cells. In their form, red blood cells are spherical with slim centers resembling a sinker while not a hole within the middle. Abnormal haemoglobin structure will, therefore, disrupt the form of red blood cells and impede their performance and flow through blood vessels. Erythropoietin (EPO), also known as erythropoetin, haematopoietin, or haemopoietin, could be a compound protein protein secreted primarily by the excretory organ in response to cellular hypoxia; it stimulates red somatic cell production (erythropoiesis) within the bone marrow. Low levels of EPO (around $10 \mathrm{mU} / \mathrm{mL}$ ) square measure perpetually secreted decent to make amends for traditional red somatic cell turnover. Common causes of cellular drive leading to elevated levels of EPO (up to $10000 \mathrm{mU} / \mathrm{mL}$ ) embrace any anemia, and hypoxemia because of chronic respiratory organ illness. Erythropoietin is created by opening fibroblasts within the excretory organ in shut association with the peritubular capillary and proximal convoluted tube. It's additionally created in perisinusoidal cells within the liver. Liver production predominates within the vertebrate and perinatal period; nephritic production predominates in adulthood. It's homologous with thrombopoietin. Preterm infants have low plasma levels of erythropoietin (EPO), providing a principle for the employment of erythropoiesis-stimulating agents (ESAs) to forestall or treat anaemia and to supply neuro protection and protection against necrotising enterocolitis (NEC). Darbepoetin (Darbe) and EPO are presently out there ESAs. To determine whether or not extremly low birth weight infants (ELBW) transfused at lower Hb thresholds versus higher thresholds have totally different rates of survival or morbidity at discharge. Red blood cell (RBC) transfusion is often considered a life-saving measure in critically ill neonates. The smallest and least mature infants tend to receive the largest amount of transfusions. RBC transfusion itself has also been suggested as an independent risk factor of poor clinical outcome in critical patients. Our aim is to study if there are associations between RBC transfusion and in-hospital mortality, short-term morbidities, and late neurodevelopmental outcome in extremely low birth weight (ELBW) preterm infants. Outcomes of extremely low gestational age neonates (ELGANs) may be adversely impacted by packed red blood cell (pRBC) transfusions. We investigated the impact of transfusions on neurodevelopmental outcome in the Preterm Erythropoietin (Epo) Neuroprotection (PENUT) Trial population. Objectives: This research is done in order to see if RBC transfusion administered to keep up low as compared to high hemoprotein thresholds reduces mortality or morbidity in terribly low birth weight infants registered at intervals 3 days of birth. Selection criteria: We tend to chosen irregular controlled trials (RCTs) scrutiny the results of early versus late, or restrictive versus liberal RBC transfusion regimes in low birth weight infants applied at intervals 3 days of birth, with mortality or major morbidity as outcomes. Methods: We performed Associate in Nursing open, multicenter trial during which infants with a birth weight of one thousand (1000)g or less and a age between twenty two weeks zero days and twenty eight weeks six days were arbitrarily allotted among forty eight hours once delivery to receive red-cell transfusions at higher or lower haemoglobin thresholds till thirty six weeks of postmenstrual age or discharge, whichever occurred initial. The first outcome was a composite of death or neurodevelopmental impairment (cognitive delay, brain disorder, or hearing or vision loss) at twenty two to twenty six months aged, corrected for immatureness. Results: A total of 1824 infants (mean birth weight, $756 \mathrm{~g}$; mean age, 25.9 weeks) underwent randomization. There was a between-group distinction of $1.9 \mathrm{~g}$ per metric capacity
\end{abstract}


unit (19 g per liter) within the pretransfusion mean haemoprotein levels throughout the treatment amount. Primary outcome information was obtainable for 1692 infants (92.8\%). Of 845 infants within the higher-threshold cluster, $423(50.1 \%)$ died or survived with neurodevelopmental impairment, as compared with 422 of 847 infants (49.8\%) within the lower-threshold cluster (relative risk adjusted for birth-weight stratum and center, 1.00; ninety fifth confidence interval [CI], 0.92 to 1.10; $\mathrm{P}=0.93)$. At 2 years, the higher- and lower-threshold teams had similar incidences of death (16.2\% and $15.0 \%$, severally) and neurodevelopmental impairment (39.6\% and $40.3 \%$, severally).

At discharge from the hospital, the incidences of survival which is not severe complications were twenty eight.5\% and $30.9 \%$, respectively. Serious adverse events occurred in twenty two.7\% and $21.7 \%$, respectively. Impact: Transfusion range, volume, and donor exposure within the time of life area unit related to worse neurodevelopmental (ND) outcome at a pair of years aged, as assessed by the Bayley baby Scales of Development, Third Edition (BSID-III). The impact of infant packed red somatic cell transfusions on the neurodevelopmental outcome of preterm infants is unknown. we tend to speculate that ways to attenuate the requirement for transfusions might improve neurodevelopmental outcomes. Conclusion: In extremely-low-birth-weight infants, the next haemoprotein threshold for red-cell transfusion failed to improve survival while not neurodevelopmental impairment at twenty two to twenty six months older, corrected for prematureness.

Keywords: ELBW, erythropoietin, packed red blood cell, haematocrit, anemia, blood transfusion.

\section{Introduction}

Packed red-cell transfusions are ordinarily wont to treat low haemoprotein levels in anemic infants in baby medical aid units (NICUs) ${ }^{[1]}$. Infants with associate extremely-low birth weight $(<1000 \mathrm{~g})$ are at high risk for anemia attributable to state, impaired organic process, and frequent blood sampling.

Thresholds for transfusion vary ${ }^{[2]}$ as a result of proof from randomised trials is proscribed ${ }^{[3-7]}$.

The biggest trial to tell our trial protocol (available with the total text of this text at NEJM.org) concerned quite 450 neonates. That trial showed no distinction between low and high haemoprotein transfusion thresholds with relation to the first outcome of clinically important complications in neonates to thirty six weeks of postmenstrual age ${ }^{[3]}$ or the chance of death or severe adverse neurodevelopmental outcomes at eighteen to twenty one month's ancient, corrected for immaturity ${ }^{[6]}$. However, a logical fallacy analysis advised that the chance of mild-to-moderate psychological feature delay was reduced with higher haemoprotein thresholds ${ }^{[6]}$.

To additional examination for the result of transfusion follow on neurodevelopment in extremely-low-birth-weight infants, we have a tendency to conducted the Transfusion of Prematures (TOP) trial to check the hypothesis that the next haemoprotein threshold for red-cell transfusions, as compared with a lower threshold, would cut back the incidence of death or neurodevelopmental impairment in infants at twenty two to twenty six months ancient, corrected for immaturity. Each transfusion algorithms utilized in the trial was in keeping with those utilized in current study practice ${ }^{[2]}$.

\section{Methods}

\section{TRIAL style AND OVERSIGHT}

This open, multicenter, randomized, controlled trial was designed by the authors and conducted in nineteen centers (41 NICUs) taking part within the babe analysis Network of the Eunice Kennedy Shriver National Institute of kid Health and Human Development (NICHD), unitedly with the National Heart, Lung, and Blood Institute (NHLBI). Infants underwent organization between December 31, 2012, and April twelve, 2017, with followup through February three, 2020.

Four of the authors utilized by RTI International, the information coordinative center for the babe analysis Network, undertook coordination, monitoring, and information assortment, storage, management, and analysis. The authors utilized by RTI International had full access to any or all the information within the trial and take responsibility for the integrity of the information and also the accuracy of the information analysis. The primary five authors and also the last author ready a draft of the manuscript that was reviewed and approved by the trial commission and every one website investigators. The NICHD and NHLBI workers had input into the trial style, conduct, analysis, and drafting of the manuscript. All the authors vouch for the accuracy and completeness of the coverage and for the fidelity of the trial to the trial protocol.

Investigators at every center (listed in Table S1 within the Supplementary Appendix, on the market at NEJM.org) and RTI International obtained approval of the institutional review board at every taking part website and supervised the consent method and trial procedures. Written consent was obtained from the parent or fiduciary of every kid. An independent that oversight was provided by the information and safety observance committee of the babe analysis Network, including consultants in pediatrics, bioethics, and biostatistics, also as a babe transfusion specialist appointed for this trial by the NHLBI.

\section{Participants}

Infants with a birth weight of a thousand $g$ or less, a fetal age between twenty two weeks zero days and twenty eight weeks halfdozen days, and a postnatal age of forty eight hours or less were eligible to participate within the trial. Infants were excluded if they were thought of to be dead by the attending neonatologist, had cyanotic inherent cardiovascular disease, had oldsters World Health Organization were critical insertion, had a parent with blood disease or inherent anemia, had received a transfusion in utero, had twin-to-twin transfusion syndrome or isoimmunolysis unwellness, or had received a previous red-cell transfusion once the primary half-dozen hours of life. different exclusion criteria were the chance that the infant's family wouldn't be able to come back for follow-up assessment at twenty two to twenty six months, receipt or planned receipt of glycoprotein, and an inherent condition (other than premature birth) adversely poignant expectancy or neurodevelopment.

\section{Randomization}

The infants were haphazardly allotted in an exceedingly 1:1 quantitative relation to the higher- or lower-threshold cluster. randomisation was performed centrally by phonephone with the information coordinative center. randomisation was stratified in keeping with birth weight ( $<750 \mathrm{~g}$ or 750 to a thousand $\mathrm{g}$ ) and trial center and balanced inside haphazardly chosen block sizes of two or four patients. Multiple-birth siblings underwent randomization one by one. 


\section{Interventions}

All routine red-cell transfusions in each team were guided by algorithms till the infants reached thirty six weeks of postmenstrual age or discharge from the NICU of the trial hospital, whichever occurred $1^{\text {st }}$ (Table S2). Transfusion algorithms were per current observe ${ }^{[2,8]}$ together with observe at taking part babe analysis Network sites. We tend to adhered to moral recommendations concerning trials of dose-adjusted therapies ${ }^{[9]}$. Hemoprotein transfusion thresholds in each teams were determined in keeping with postnatal age (highest within the 1st week of life, lower in every of the two consecutive weeks, and stable thereafter) and in keeping with the utilization of metastasis support (a higher threshold once metastasis support was warranted).

Metastasis support was outlined as mechanical ventilation, continuous positive airway pressure, a fraction of impressed chemical element (Fio2) bigger than 0.35 , or delivery of chemical element or space air by nasal tube at a flow of one liter per minute or even more.

With excellent adherence to those transfusion algorithms throughout the treatment amount, the mean between-group distinction within the pretransfusion hemoprotein levels was expected to be 2.0 to $2.5 \mathrm{~g}$ per metric capacity unit (20 to twenty five $\mathrm{g}$ per liter). The transfusion volume was fifteen cubic centimeter per metric weight unit of weight. All transfusions received by the infants were from $\mathrm{ABO}$-compatible and $\mathrm{Rh}$ compatible donors. The clinicians were allowable to deviate from the applicable algorithmic rule quickly for reasons laid out in the protocol (e.g., if the child had haemorrhage or anticipated haemorrhage throughout or once surgery, severe hypoxemia, cardiovascular disease, or sepsis). All the blood transfusions were recorded and centrally reviewed for consistency with the algorithms; if they were inconsistent, they were deemed to be deviations. All deviations were severally adjudicated as being either even by the protocol or violations in keeping with agreement of two investigators UN agency were unaware of the treatment assignments.

\section{Primary Outcome}

The primary outcome was a composite of death or neurodevelopmental impairment in infants at twenty two to twenty six months more matured, corrected for immatureness. All the follow-up examiners, together with psychologists and psychometrics, were unaware of the treatment assignments.

Neurodevelopmental impairment was outlined collectively or a lot of the subsequent components: psychological feature delay, moderate or severe brain disease, or severe vision or hearing disorder. Psychological feature delay was outlined as a composite psychological feature score of but eighty five (1 Mount Rushmore State below the mean of 100) on the Bayley Scales of kid and tike Development, third edition10; scores vary from fifty five to one hundred forty five, with higher scores indicating higher performance. Moderate brain disease was outlined as level II or III within the Gross Motor perform organisation (GMFCS) (levels vary from I [mild impairment] to $\mathrm{V}$ [most severe impairment]), and severe brain disease as GMFCS levels IV or V.11 Severe vision loss was outlined as a corrected sight within the higher eye of but $20 / 200$. Severe disability was outlined as bilateral hearing disorder that hearing aids or tube-shaped structure implants were secure, in line with a hearing assessment conducted by sound field testing or in line with testing of audile brain-stem responses.

We have a tendency to prespecified that infants while not moderate or severe brain disease and while not vision or hearing disorder had to finish the psychological feature subtest of the
Bayley Scales with success so as to be counted as intact for the composite primary outcome.

\section{Secondary Outcomes}

Table S3 lists all the prespecified secondary outcomes to 1st discharge home and at a pair of years ancient. Prespecified secondary outcomes at twenty two to twenty six months ancient enclosed death, neurodevelopmental impairment and its four parts, and a lot of careful analyses of the composite psychological feature, language, and motor scores on the Bayley Scales. When a protocol amendment in 2013, the fogeys or guardians of the infants completed the kid Behavior Checklist ${ }^{[12]}$ rather than the transient Infant-Toddler Social and Emotional Assessment so as to accommodate revised babe analysis Network follow-up procedures.

Prespecified secondary babe outcomes enclosed survival to initial hospital discharge while not severe complications. These complications enclosed grade three or four cavity hemorrhage, cystic periventricular leukomalacia, or ventriculomegaly diagnosed with the utilization of clinically performed ultrasonographic examination of the head ${ }^{[13]}$; stage three or larger retinopathy of immaturity or receipt of treatment for that condition ${ }^{[14]}$; associate degreed bronchopulmonary abnormal condition diagnosed at thirty six weeks of postmenstrual age on the premise of a documented would like for supplemental chemical element (defined as an Fio2 of $\geq 0.30$ or inability to pass the chemical element reduction take a look at if the Fio2 was 0.22 to 0.29$)^{[15]}$. Stage a pair of or three inflammatory disease was recorded ${ }^{[16]}$. The infant's head circumference, weight, and length at a postmenstrual age of thirty six weeks, postmenstrual age at the last use of caffein medical care, and $\{$ also the $\}$ variety of packed red-cell transfusions were also secondary outcomes. for every transfusion, it had been noted whether or not the administration was in keeping with the trial protocol.

\section{Statistical Analysis}

Our main objective was to assess the between-group distinction within the composite primary outcome of death or neurodevelopmental impairment at twenty two to twenty six months ancient, corrected for immaturity. On the premise of a previous trial, ${ }^{[6]}$ we have a tendency to hypothesized that there would be associate degree absolute between-group distinction of seven share points within the incidence of death or neurodevelopmental impairment. Given a fifty two incidence of death or neurodevelopmental impairment (unpublished data) within the babe analysis Network in 2005-2008 (with entry criteria that were just like those during this trial), we have a tendency to assumed outcome rates of fifty three.5\% for the lower-threshold cluster and forty six. 5\% for the higher-threshold cluster targeted around a conservative overall event rate of fifty within the 2 treatment teams. We have a tendency to calculable that with a sample of 1824 infants and 100 percent loss to follow-up, the trial would have eightieth power to sight associate degree absolute distinction of seven share points within the incidence of the first outcome between the trial teams, at a two-tailed kind I error rate of 0.05 .

The independent information and safety watching committee reviewed the incoming information at four time points throughout the enrollment amount, as prespecified by the trial protocol. As a result of primary outcome information were offered solely at twenty two to twenty six months of follow-up, applied mathematics interim watching at four intervals targeted on a composite safety outcome of in-hospital death, inflammatory disease, or adverse findings on ultrasonographic examination of the 
top. The importance of the interim safety associate degreealyses with O'Brien-Fleming boundaries was calculated with a LanDeMets defrayal performs to preserve an overall kind I error rate of 0.05 for the composite safety outcome.

All the analyses were adjusted for stratification in keeping with birth-weight cluster and center, and every one the infants were evaluated in keeping with treatment assignment. The first outcome was analyzed with sturdy Poisson regression to get adjusted relative risks and ninety fifth confidence intervals. The consistency of the treatment impact across birth-weight strata and sexes and treatment heterogeneousness across the varied centers were examined by adding appropriate interaction terms to the adjusted models. Binary secondary outcomes, as well as red-cell transfusion outcomes, were analyzed with the utilization of strong Poisson regression. Regression was used for the amount of transfusions received. different secondary outcomes, like the time to regain birth weight and time to full enteral feeding, were analyzed with Cox proportional-hazards survival regression, and information were expurgated for deaths. There was no prespecified attempt to regulate for multiplicity of testing for the secondary outcomes, and a $\mathrm{P}$ worth is reportable just for the first outcome (with a two-sided $\mathrm{P}$ worth $<0.05$ thought-about to point applied mathematics significance); all the opposite analyses area unit explorative. Analyses were conducted with the utilization of software SAS, version 9.4 (SAS Institute).

\section{Results}

\section{Patients}

The composite primary outcome at twenty two to twenty six months elderly can be determined for 1692 of the 1824 infants registered $(92.8 \%)$. See (Figure.1).

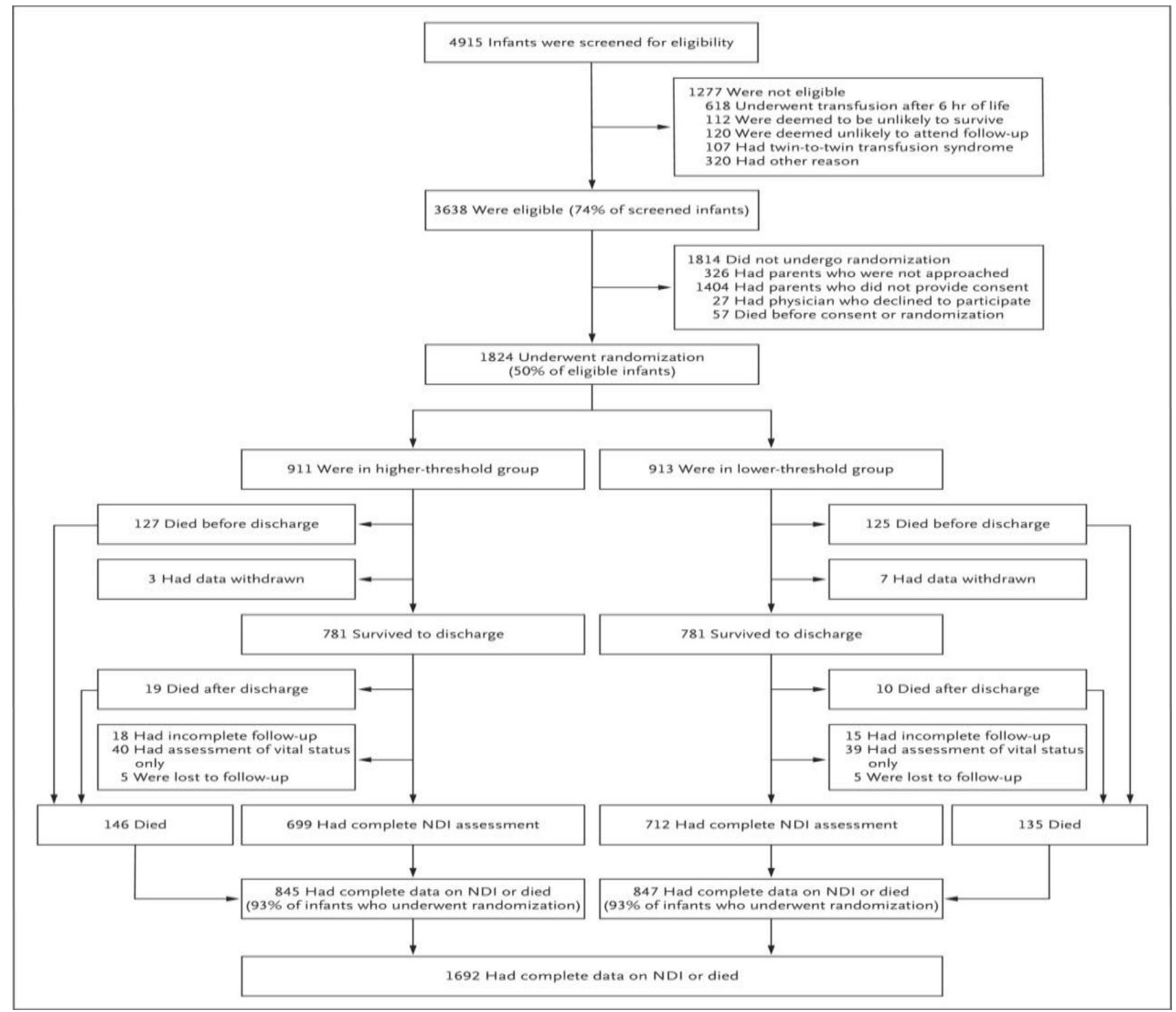

Figure 1: Screening, organisation, (Intervention, and Follow-up)

Infants were screened for eligibility on condition that they met the inclusion criteria of a birth weight of one thousand $g$ or less, a age between twenty two weeks zero days and twenty eight weeks half dozen days, admission to the middle baby medical care unit among forty eight hours once birth, and therefore the ability to bear organisation among forty eight hours once birth. The assigned intervention was to increase till thirty six weeks of postmenstrual age, unless the child had been transferred or discharged before that age. Within the higher-threshold cluster, 591 infants completed the treatment per protocol up to thirty six weeks, and an extra 137 did thus with one or a lot of protocol violations, whereas 183 interrupted treatment early thanks to death, withdrawal, or transfer or discharge. Within the lower-threshold cluster, 562 infants completed the treatment per protocol up to thirty six weeks, an extra 186 did thus with one or a lot of protocol violations, and one hundred sixty five interrupted treatment before thirty six weeks. 
Within the higher-threshold group, twenty three infants withdrew from the intervention early, of which three had parents who didn't consent to their inclusion in future analyses. Within the lowerthreshold cluster, sixteen infants withdrew from the intervention early; of which seven had parents who didn't consent to their inclusion in future analyses. 2 infants who had died before thirty six weeks of postmenstrual age additionally withdrew however during this figure they were counted as infants World Health Organization died. Infants with incomplete follow-up enclosed those that given for the follow-up examination however were missing a key element, sometimes the psychological feature subtest of the Bayley Scales of child and nipper Development assessment (14 infants within the higher-threshold cluster and eight infants within the lower-threshold group), and people who didn't go for examination however had parents who provided restricted form information (4 and seven infants, respectively). NDI denotes neurodevelopmental impairment.

Selected characteristics of those infants and their mothers area unit shown in Table one, beside those of the whole trial cohort, in step with treatment cluster. All the baseline characteristics of the 911 infants who were willy-nilly appointed to the higher-threshold cluster were kind of like those of the 913 infants who were appointed to the lower-threshold group, and these characteristics were similar within the 2 teams of infants within the end cohort for the first outcome.

\section{SEPARATION OF THE hemoprotein LEVELS AND PROTOCOL VIOLATIONS}

At the time of randomisation, the mean $( \pm \mathrm{SD})$ hemoprotein levels were similar within the 2 teams $(13.8 \pm 2.6 \mathrm{~g}$ per decilitre within the higher-threshold cluster and thirteen. $7 \pm 2.6 \mathrm{~g}$ per decilitre within the lower-threshold group) (Table 1). Later on, the pretransfusion mean hemoprotein levels differed between the teams by one. $9 \mathrm{~g}$ per decilitre $(19 \mathrm{~g}$ per liter) throughout the treatment amount $(\mathrm{P}<0.001)$ (Figure 2A). A comparison of all hemoprotein levels obtained throughout the infants' hospital keep confirmed separation between the mean hemoprotein levels within the 2 teams see (Figure 2B). Of all red-cell transfusions, $3.5 \%$ were adjudicated to be protocol violations, and $2.5 \%$ of all the transfusions mandated by the various transfusion algorithms weren't administered. see (Table S5).

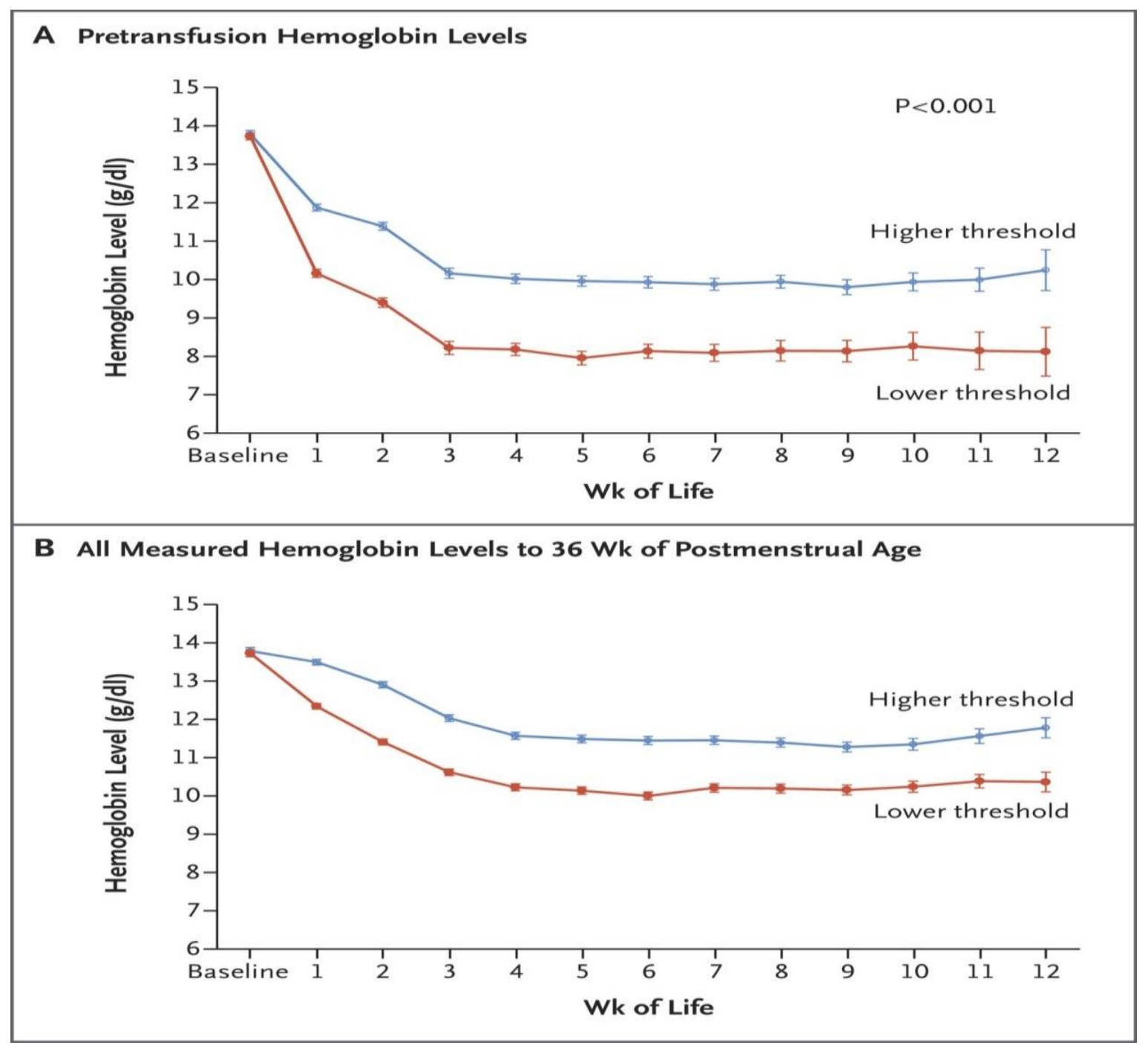

Figure 2: Separation of haemoglobin Levels between the Treatment teams.

Haemoglobin levels within the higher-threshold and lowerthreshold teams were recorded before enrollment and till thirty six weeks of postmenstrual age. Values square measure suggests that and ninety fifth confidence intervals (indicated by I bars), adjusted for kid as a random impact. Haemoglobin tests were performed at clinical discretion and weren't set by protocol. Panel shows the haemoglobin levels that prompted a red-cell transfusion. Panel B shows all haemoglobin levels that were measured within the 2 teams throughout the treatment amount. 


\section{Primary Outcome}

Complete information for the first outcome was on the market for 1692 of 1824 infants (92.8\%). Of the 845 infants with information for this outcome who were appointed to the upper transfusion threshold, $423(50.1 \%)$ died or survived with impairment, as compared with 422 of 847 infants $(49.8 \%)$ appointed to the lower transfusion threshold (adjusted relative risk, 1.00; ninety fifth confidence interval $[\mathrm{CI}], 0.92$ to $1.10 ; \mathrm{P}=0.93$ ). Similar results were obtained in an exceedingly post hoc ergo propter hoc sensitivity analysis during which a generalized estimating equation was wont to account for any correlation among siblings of a multiple birth (adjusted relative risk, $1.01 ; 95 \% \mathrm{CI}, 0.92$ to 1.10 ; $\mathrm{P}=0.88$ ). The incidences of all elements of the first outcome were similar within the 2 teams (Table 2).

Additionally, there was no proof that the consequences of the transfusion strategy on the first outcome differed in step with center, birth-weight cluster, or sex (Fig. S1). In sensitivity analyses accounting for missing primary outcome information, the results were materially unchanged, despite whether or not all missing outcomes were assumed to be events (adjusted relative risk, 1.01; $95 \% \mathrm{CI}, 0.93$ to 1.09 ) or nonevents (adjusted relative risk, 1.00; $95 \%$ CI, 0.91 to 1.10$)$.

Table 2: Primary Composite Outcome and Components of the Primary Composite Outcome at 2 Years

\begin{tabular}{|l|l|l|l|l|}
\hline Outcome & $\begin{array}{l}\text { Higher Hemoglobin } \\
\text { Threshold (N=845) }\end{array}$ & $\begin{array}{l}\text { Lower Hemoglobin } \\
\text { Threshold (N=847) }\end{array}$ & $\begin{array}{l}\text { Adjusted Relative } \\
\text { Risk (95\% CI) }\end{array}$ & $\begin{array}{l}\text { P } \\
\text { Value }\end{array}$ \\
\hline & \multicolumn{2}{|l|}{ no. of infants/total no. (\%) } & & \\
\hline Primary outcome: death or neurodevelopmental impairment & $423 / 845(50.1)$ & $422 / 847(49.8)$ & $1.00(0.92-1.10)$ & 0.93 \\
\hline Components of primary outcome & & & & \\
\hline Death & $146 / 903(16.2)$ & $135 / 901(15.0)$ & $1.07(0.87-1.32)$ & \\
\hline Neurodevelopmental impairment & $277 / 699(39.6)$ & $287 / 712(40.3)$ & $1.00(0.88-1.13)$ & \\
\hline Cognitive delay & $269 / 695(38.7)$ & $270 / 712(37.9)$ & $1.04(0.91-1.18)$ & \\
\hline Moderate or severe cerebral palsy & $48 / 711(6.8)$ & $55 / 720(7.6)$ & $0.87(0.60-1.26)$ & \\
\hline Severe vision impairment & $5 / 713(0.7)$ & $6 / 720(0.8)$ & $0.83(0.25-2.76)$ & \\
\hline Severe hearing impairment & $14 / 710(2.0)$ & $25 / 715(3.5)$ & $0.56(0.29-1.07)$ & \\
\hline
\end{tabular}

The relative risk was adjusted for birth-weight and center stratum. The $\mathrm{P}$ value is reported only for the composite primary outcome. For the secondary outcomes, $95 \%$ confidence intervals (CIs) were not adjusted for multiplicity and should not be used to infer definitive treatment effects. Infants with incomplete follow-up but with known vital status were included in these numbers. Five infants in each group had unknown vital status at the start of the 2year follow-up window.

Cognitive delay was defined as a composite cognitive score of less than 85 (1 SD below the mean of 100) on the Bayley Scales of Infant and Toddler Development (BSID-III), third edition; scores range from 55 to 145 , with higher scores indicating better performance ${ }^{[10]}$. Moderate or severe cerebral palsy was defined as a Gross Motor Function Classification System level II or higher (levels range from I [mild impairment] to $\mathrm{V}$ [most severe impairment]).

Level II denotes moderate cerebral palsy with a limited ability to walk. Levels III to V indicate increasing severity of gross motor impairment.

Trial centers with low incidences of blindness or hearing impairment were pooled with their nearest geographic center before estimation of adjusted relative risks.

\section{Secondary Outcomes}

The incidences of prespecified short-run and long secondary outcomes were similar among infants within the higher-threshold cluster and people within the lower-threshold group (Table 3). The mean $( \pm \mathrm{SD})$ variety of transfusions was half-dozen.2 \pm 4.3 and $4.4 \pm 4.0$, severally (mean distinction, $1.71 ; 95 \% \mathrm{CI}, 1.37$ to 2.05 ) (Table 3). A complete of 885 of 911 infants (97.1\%) within the higher-threshold cluster and 804 of 913 infants $(88.1 \%)$ within the lower-threshold cluster received a minimum of one transfusion (adjusted relative risk, 1.10; 95\% CI, 1.08 to 1.13). further transfusion information area unit provided in Table S6. Results for alternative prespecified and post hoc ergo propter hoc secondary outcomes area unit provided in Tables S7 and S8. Serious adverse events occurred in twenty two.7\% of the infants within the higherthreshold cluster and twenty one.7\% of these within the lowerthreshold cluster (adjusted relative risk, $1.04 ; 95 \% \mathrm{CI}, 0.88$ to 1.23 ) (Table S9).

Plus-minus values square measure means that \pm SD. Outcomes to hospital discharge square measure reported for 908 infants within the higher-threshold cluster and 906 infants within the lower-threshold group. These varietys were calculated because the number of infants in every group who underwent randomisation (911 and 913, respectively), minus the quantity of infants in every cluster (3 and seven, respectively) for whom consent for knowledge assortment was withdrawn. Outcomes at two years square measure reported for 699 infants within the higher-threshold cluster and 712 infants within the lower-threshold cluster (some of the denominators shown square measure larger than the quantity of patients World Health Organization had an entire follow-up assessment as a result of partial knowledge from incomplete assessments were included). Estimates square measure adjusted for birth-weight and center stratum. IQR denotes interquartile vary.

For categorical outcomes, adjusted variations square measure adjusted relative risks, with the lower-threshold group because the reference. For time to full enteral feeding, variations square measure adjusted hazard ratios; for the remaining continuous outcomes, they're adjusted mean variations. The ninety fifth confidence intervals don't seem to be adjusted for multiplicity and may not be accustomed infer definitive treatment effects. 
Table 3: Prespecified Secondary Outcomes to Hospital Discharge and at 2 Years.

Table 3. Prespecified Secondary Outcomes to Hospital Discharge and at 2 Years."

\section{Outcome}

Outcomes to hospital discharge

Survival to discharge without severe complications - no./total no. $(\%) \div$

Bronchopulmonary dysplasia at 36 wk postmenstrual age - no./total no. (\%)

Retinopathy of prematurity stage $\geq 3$ or treatment for that condition received - no./total no. (\%) 9

Grade 3 or 4 intraventricularhemorrhage, cystic periventricular leu. komalacia, or ventriculomegaly diagnosed on ultrasonographic examination - no./total no. (\%)

Necrotizing enterocolitis, Bell's stage $22-$ no./total no. (\%) $\mid$

No. of transfusions per infant

Anthropometric measures ${ }^{\text {wi }}$

Weight for age

$$
\text { No. of infants }
$$

Change in z score

Length for age

No, of infants

Change in $z$ score

Head circumference for age

No, of infants

Change in z score

Postmenstrual age at final tracheal extubation in infants who were intubated

No. of infants

Wh

Postmenstrual age at final caffeine dose in infants who received caffeine treatment

No, of infants

Wh

Length of hospital stayt

No. of infants

Median days (IQR)

Time to full enteral feeding

No. of infants

Median days (IQR)

96 (72 to 129)

19.5 (14 to 29$)$

$20 / 710(2.8)$

$248 / 715(34.7)$

Respiratory disease necessitating readmission before follow.up

Developmental delay assessed with BSID.IIIt个†

Composite language score $<85$

Composite motor score $<85$

Composite cognitive score $<70$

Composite language score $<70$

Composite motor score $<70$
769

$=1.2 \pm 0.8$

715

$-1.9 \pm 1.0$

754

$-1,4 \pm 1,0$

796

$30.1 \pm 3.4$

$30.2 \pm 3$

$-0.11(-0.43$ to 0.21$)$

882

$33,8+3,0$

887

$34,0 \div 2.8$

$-0.19(-0.45$ to 0.07$)$

908

808

$20 / 717(2.8)$

$61 / 700(8.7)$

$42 / 714(5.9)$

Adjusted Relative Risk, Hazard Ratio, or (95\% CI) $\hat{~}$

$0.93(0.81$ to 1.06$)$

$.14(0.94$ to 1.39$)$

$0.94(0.77$ to 1.16$)$

$0.95(0.73$ to 1.25$)$

$1.71(1.37$ to 2.05$)$

$0.04(-0.04$ to 0.11$)$

$0.07(-0.01$ to 0.16$)$

$-0.01(-0.10$ to 0.08$)$

$-1.25(-6.96$ to 4,48$)$

$19.0(15$ to 30$) \quad 0.96(0.87$ to 1.05$)$

$11 / 720(1.5) \quad 1.85(0.90$ to 3.82$)$

$22 / 728(3.0) \quad 0.92(0.51$ to 1.67$)$

$52 / 710(7.3) \quad 1.17(0.83$ to 1.66$)$

$41 / 726(5.6) \quad 1.04(0.68$ to 1.57$)$

$230 / 726(31.7) \quad 1.09(0.95$ to 1.26$)$

\begin{tabular}{rrl}
$355 / 671(52.9)$ & $368 / 691(53.3)$ & $1.01(0.91$ to 1.11$)$ \\
$255 / 678(37.6)$ & $280 / 695(40.3)$ & $0.96(0.84$ to 1.09$)$ \\
$88 / 695(12.7)$ & $96 / 712(13.5)$ & $0.96(0.74$ to 1.25$)$ \\
$164 / 671(24.4)$ & $163 / 691(23.6)$ & $1.06(0.88$ to 1.27$)$ \\
$87 / 678(12.8)$ & $99 / 695(14.2)$ & $0.91(0.70$ to 1.18$)$ \\
\hline
\end{tabular}

Bronchopulmonary abnormality was diagnosed on the idea of the requirement for supplemental gas once an identical gas reduction check at thirty six weeks of postmenstrual age.

$1.01(0.91$ to 1.11$)$

$0.96(0.74$ to 1.25$)$

$6(0.88$ to 1.2$)$

$87 / 678(12.8) \quad 99 / 695(14.2) \quad 0.91(0.70$ to 1.18$)$
Severe complications were outlined as bronchopulmonary abnormality, retinopathy of immatureness of stage three or higher or that treatment was bonded, Associate in Nursing an adverse finding on ultrasonographic examination of the top. 
Retinopathy of immatureness was recorded for infants who underwent a retinal examination before discharge from the infant medical aid unit.

Bell's stages vary from one to three, with higher stages indicating larger severity of illness.

$\mathrm{Z}$ scores square measure derived from Olsen growth curves

Length of keep was up to hospital discharge or death, whichever occurred 1 st.

A complete of 808 of 907 infants $(89.1 \%)$ within the higher-threshold group and 824 of 906 infants $(90.9 \%)$ within the lower-threshold cluster earned full enteral feeding throughout the amount of observation. The hazard quantitative relation is also taken because the odds of accomplishing full enteral feeding quicker at any purpose in time. Deaths were thought-about to be a censoring event.

Severe encephalopathy was outlined as Gross Motor operate arrangement (GMFCS) levels IV or V on Associate in
Nursing ordinal scale on that levels vary from I (mild impairment) to $\mathrm{V}$ (most severe impairment).

The trial centers with low incidences of this condition were pooled with their nearest geographic center.

Abnormal condition was outlined as a head circumferencefor-age z score of but 2, per growth curves developed by the world Health Organization (WHO) (WHO Multicentre Growth Reference Study cluster, Geneva; World Wide Web.who.int/toolkits/childgrowth-standards/standards/head-circumference-for-age. opens in new tab) in infants in whom age was corrected for immatureness.

A seizure disorder was outlined as a report of getting one or a lot of seizures since discharge or of standard use of anticonvulsants or seizure medications.

BSID-III composite language and motor scores range from 40 to 160 and BSID-III composite cognitive scores range from 55 to 145 , with higher scores indicating better performance ${ }^{[10]}$. Composite BSID-III scores of less than 85 are less than $1 \mathrm{SD}$ below the mean of 100. Composite BSID-III scores of less than 70 are less than 2 SD below the mean of 100. See (Figure.3)

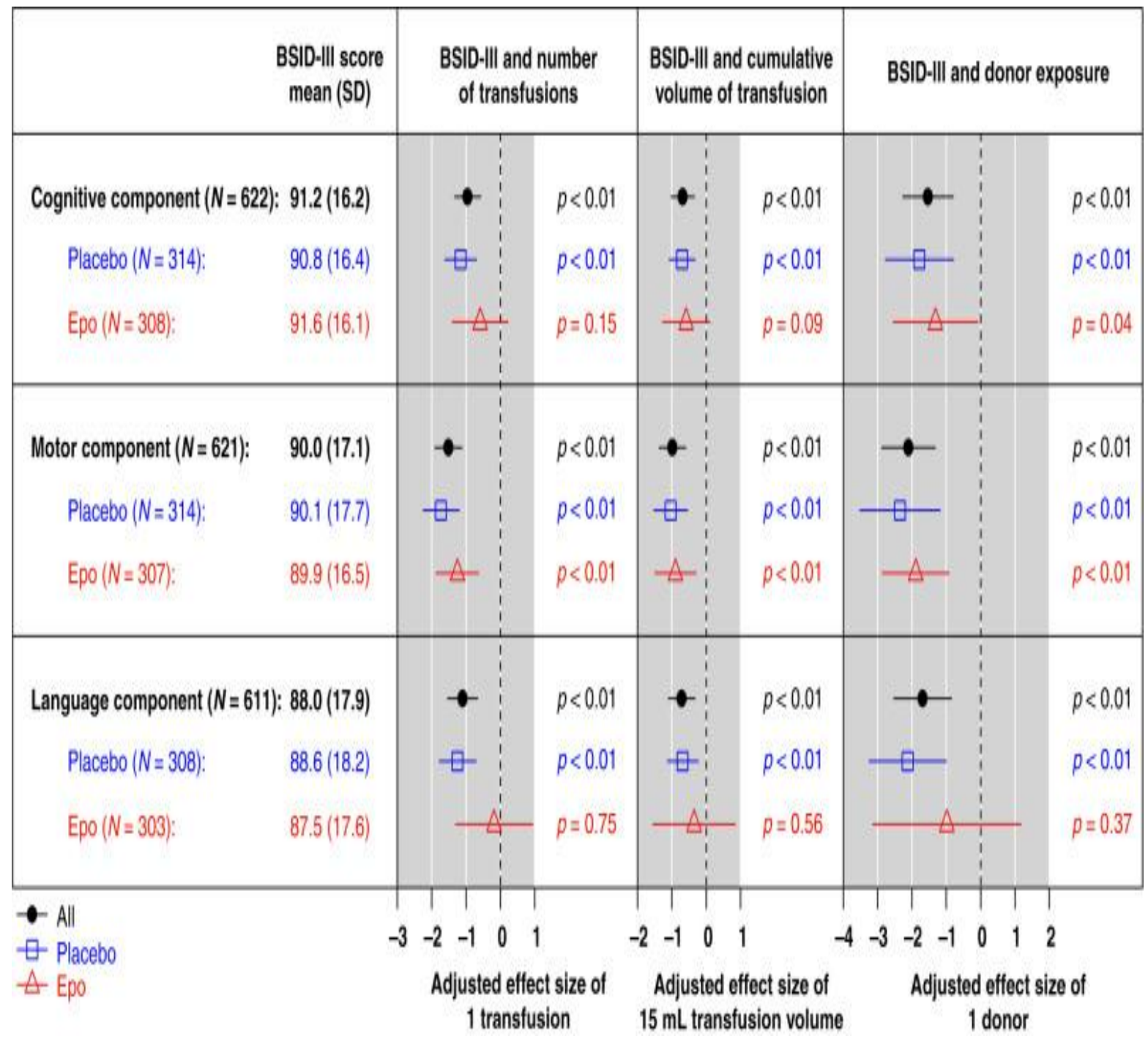

Figure.3 shows: Primary analysis of BSID-III element scores and pRBC transfusion exposures.

The associations between mean BSID-III element scores (cognitive, motor, and language) and pRBC transfusion exposures (number of transfusions, additive volume of transfusion, and donor exposure) were examined victimisation GEE models clump on same-birth siblings, adjusted for mounted effects of treatment group, fertilization age cluster, enlisting website, and alternative potential confounders at baseline. These associations were additionally evaluated for every treatment cluster victimisation similar GEE models. The forest plot shows the calculable impact sizes of transfusion exposures on mean BSID-III scores and corresponding ninety fifth CIs. See (Figure.4) 


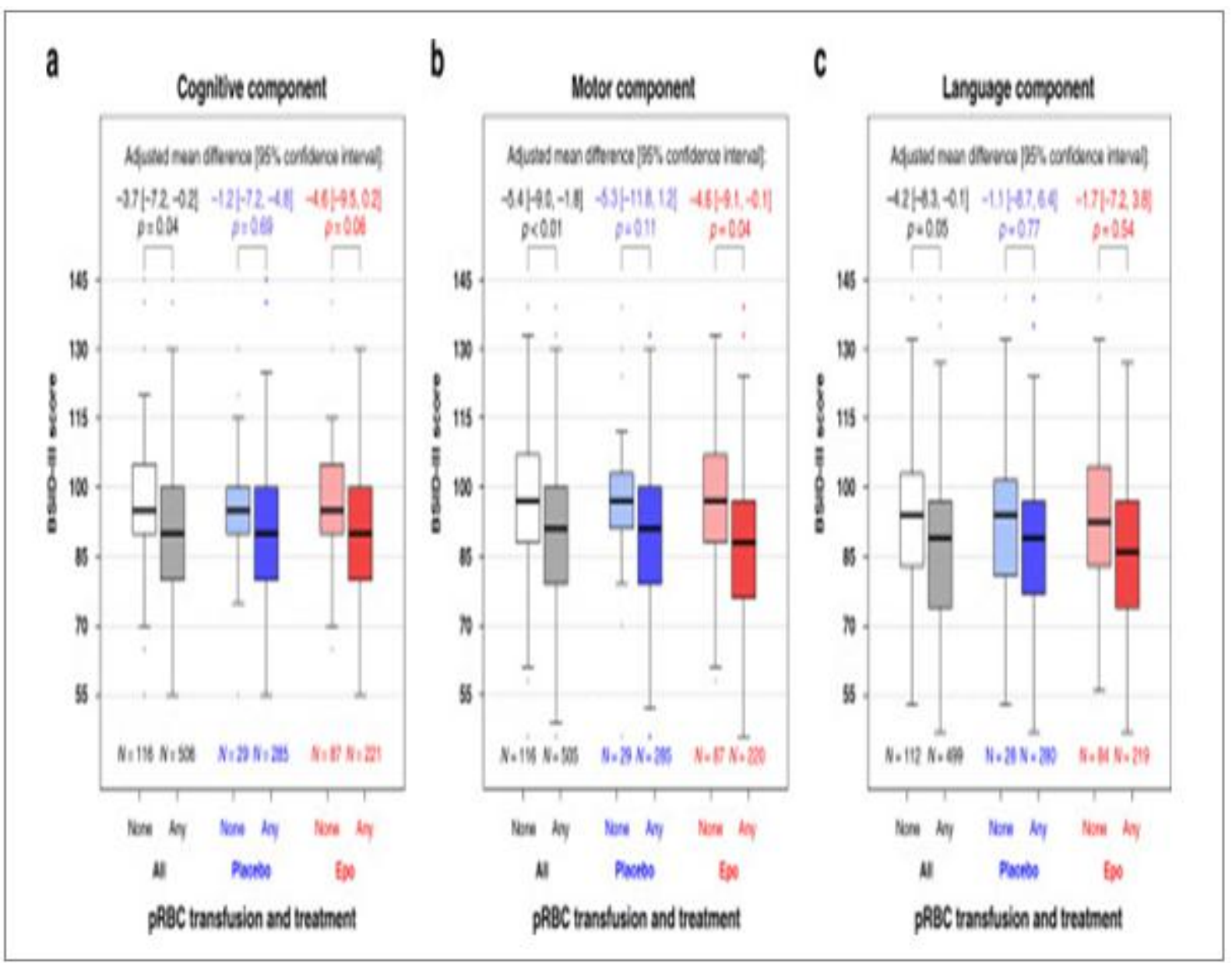

Figure 4: Shows BSID-III element scores and any pRBC transfusion.

\section{The distributions of BSID-III}

a. Psychological feature scoBSID-III element scores and any pRBC transfusion. The distributions of BSID-III a psychological feature scores,

b. Motor scores, and c language scores were summarized for UN agency those that people who were transfusionfree and who had $\geq 1 \mathrm{pRBC}$ transfusion, overall and by treatment group. the associations among all participants were examined exploitation GEE models cluster on same-birth siblings, adjusted for fastened effects of treatment cluster, fertilization age group, accomplishment website, and alternative potential contradictory variables at baseline. These associations were additionally evaluated for every treatment group exploitation similar GEE models.res, b motor scores, and c Language scores were summarized for UN agency those that people who were transfusion-free and who had $\geq 1 \mathrm{pRBC}$ transfusion, overall and by treatment cluster. the associations among all participants were examined exploitation GEE models cluster on same-birth siblings, adjusted for fastened effects of treatment cluster, fertilization age group, accomplishment site, and alternative potential contradictory variables at baseline. These associations were additionally evaluated for every treatment group exploitation similar GEE models.

\section{Discussion}

Our trial showed that among extremely-low-birth-weight infants, the chance of death or neurodevelopmental impairment at twenty two to twenty six months ancient, corrected for immaturity, wasn't considerably lower with better haemoglobin transfusion strength than with lower haemoglobin transfusion strength throughout the initial hospital course. though a logical fallacy analysis of a previous trial had advised a moderate psychological feature profit at eighteen to twenty-one months ancient in infants WHO were every which way appointed to take care of a better haemoglobin level, ${ }^{[6]}$ this, a lot of larger trial showed no proof to support associate degree improvement during this or different elements of the composite primary outcome or in the other clinically vital outcome, whether or not measured throughout the initial hospital course or at twenty-two to twenty-six months ancient. These results area unit in keeping with the failure of glycoprotein to enhance psychological feature or different neurodevelopmental outcomes despite increasing red-cell mass ${ }^{[8]}$. Our findings area unit in keeping with the results of the same however smaller European trial that were printed whereas our manuscript was beneath review [19].

Although methods to individualize red-cell transfusions with the employment of physiological measures area unit promising, more testing is required ${ }^{[20]}$. Correspondingly, the employment of transfusion in NICUs remains high. A Canadianwide study conducted in 2010-2012 showed that eighty two of infants with a birth weight between 501 and $750 \mathrm{~g}$ received red-cell transfusions ${ }^{[21]}$. As in our trial, many teams in Europe ${ }^{[22]}$ and North America ${ }^{[23]}$ have shown that the employment of transfusions decreases once a strict guideline is adopted. Within the intervention within the current trial algorithmic rule, we tend to selected haemoglobin levels that will stay among clinically accepted ranges $[2,8,9]$. 
Previous empiric knowledge on preterm infants has advised risks related to blood transfusions. an oversized empiric cohort study in Brazil involving very-low-birth-weight infants showed associate degree excess additive hazard magnitude relation for death in those that received blood transfusions, ${ }^{[24]}$ however this observation could are explained by the exaggerated severity of illness in these infants ${ }^{[25]}$. Some empiric studies have shown associate degree association between transfusions and NEC, however empiric knowledge are inconsistent, and this finding has not been supported by offered knowledge from randomised trials ${ }^{[26-30]}$. One prospective study showed that NEC wasn't related to transfusion and was additional doubtless to occur once nadir haemoglobin levels attenuated to below eight g per metric capacity unit $\left(80 \mathrm{~g}\right.$ per liter) before transfusion ${ }^{[30]}$. In different empiric studies, transfusions are joined to retinopathy of immaturity, ${ }^{[31]}$ bronchopulmonary abnormal condition, [32] and cavity haemorrhage ${ }^{[33]}$. In distinction, different studies have advised that the risks of hypoxemia and symptom of immaturity area unit exaggerated among infants WHO don't receive transfusions ${ }^{[34,35]}$. we tend to recorded the postmenstrual age at the last use of alkaloid medical aid as a proxy for symptom, and also the results were similar within the two threshold teams. we tend to found no different effects of transfusions on useful or adverse outcomes, as well as stage two or three NEC. Such discrepancies between associations shown in empiric studies and our findings underscore the pitfalls of empiric studies ${ }^{[36,37]}$. Though our trial wasn't supercharged to deal with these individual adverse outcomes, it had been an oversized trial comparison high transfusion thresholds with low transfusion thresholds during this vulnerable population.

Our trial has some vital limitations. Dazzling of the trial intervention wasn't possible at the side. However, follow-up examiners were unaware of the treatment assignments. For moral reasons, we tend to couldn't withhold nonalgorithmic transfusions (i.e., people who weren't performed per the every which way appointed transfusion algorithm), thus there was associate degree unbalanced violation rate, with additional nonalgorithmic transfusions within the lower-threshold cluster.

This imbalance presumptively mirrored the unease of some physicians with haemoglobin levels within the lower vary. Yet, the incidence of violations was low and failed to preclude sensible between-group separation in mean haemoglobin levels. Blood banks at totally different centers failed to have uniform practices; it might not are possible to manage this pragmatic multicenter trial with the employment of one bank. However, this variation is unlikely to own affected outcomes, since randomisation was stratified per center. Moreover, excluding leukoreduction, ${ }^{[38]}$ different blood-banking practices like transfusing solely recent red cells ${ }^{[39,40]}$ haven't been shown to own useful effects. The same old clinical variation in blood-banking practices enhances the generalizability of our findings.

In our trial, the next hemoprotein threshold for transfusion was related to a rise within the range of transfusions administered. However, it failed to improve survival while not neurodevelopmental impairment at twenty-two to twenty-six months getting on among extremely-low-birth-weight infants. The views expressed during this article area unit those of the authors and don't essentially represent the official views of the Eunice Kennedy Shriver National Institute of kid Health and Human Development (NICHD), the National Institutes of Health (NIH), the Department of Health and Human Services, or the U.S. government.

\section{List of abbreviations}

BT: Blood Transfusion.

ELBW: Extremely Low Weight.

Hg: Haemoglobin.

EPO: Erythropoietin.

NEC: Ecrotising Enterocolitis.

RBCs: Reed Blood Cells.

GMFCS: Gross Motor Operates Arrangement.

NIH: National Institutes of Health.

NICHD: National Institute of kid Health and Human Development.

BSID: Bayley Scales of Infant and Toddler Development.

SD: Standard Deviation.

TOP: Transfusion of Prematures.

\section{Authors' contributions}

Zaki Ghali Alhothali: have made substantial contributions to conception and design, or acquisition of data, or analysis and interpretation of date have been involved in drafting the manuscript or revising it critically for important intellectual content

Abdulrahman Salem Altalhi: have given final approval of the version to be published agree to be accountable for all aspects of the work in ensuring that questions related to the accuracy or integrity of any part of the work are appropriately investigated and resolved.

Nemer Khidhran Alghamdi: have given final approval of the version to be published agree to be accountable for all aspects of the work in ensuring that questions related to the accuracy or integrity of any part of the work are appropriately investigated and resolved.

Naif Mohammed Althobaiti: have given final approval of the version to be published agree to be accountable for all aspects of the work in ensuring that questions related to the accuracy or integrity of any part of the work are appropriately investigated and resolved.

Anas Mohammed Alqadi: have given final approval of the version to be published agree to be accountable for all aspects of the work in ensuring that questions related to the accuracy or integrity of any part of the work are appropriately investigated and resolved

Hassan Ahmed Hassan Almalki: have given final approval of the version to be published agree to be accountable for all aspects of the work in ensuring that questions related to the accuracy or integrity of any part of the work are appropriately investigated and resolved.

Raniyah Embarak D Alharbi: analysis and interpretation of date have been involved in drafting the manuscript or revising it critically for important intellectual content have given final approval of the version to be published agree to be accountable for all aspects of the work

\section{Acknowledgments}

To our beloved fathers and mothers, you were the primary motivators to finish during the short time we spent while working this paper, thank you so much. our wife or husband, professors' mate who supervised thank you very much for your support. 
[1] Patel RM, Meyer EK, Widness JA. Research opportunities to improve neonatal red blood cell transfusion. Transfus Med Rev 2016;30:165-173.

[2] Guillén U, Cummings JJ, Bell EF, et al. International survey of transfusion practices for extremely premature infants. Semin Perinatol 2012;36:244-247.

[3] Kirpalani H, Whyte RK, Andersen C, et al. The Premature Infants in Need of Transfusion (PINT) study: a randomized, controlled trial of a restrictive (low) versus liberal (high) transfusion threshold for extremely low birth weight infants. J Pediatr 2006;149:301-307.

[4] Bell EF, Strauss RG, Widness JA, et al. Randomized trial of liberal versus restrictive guidelines for red blood cell transfusion in preterm infants. Pediatrics 2005;115:16851691.

[5] Whyte R, Kirpalani H. Low versus high haemoglobin concentration threshold for blood transfusion for preventing morbidity and mortality in very low birth weight infants. Cochrane Database Syst Rev 2011;11:CD000512-CD000512.

[6] Whyte RK, Kirpalani H, Asztalos EV, et al. Neurodevelopmental outcome of extremely low birth weight infants randomly assigned to restrictive or liberal hemoglobin thresholds for blood transfusion. Pediatrics 2009;123:207-213.

[7] Nopoulos PC, Conrad AL, Bell EF, et al. Long-term outcome of brain structure in premature infants: effects of liberal vs restricted red blood cell transfusions. Arch Pediatr Adolesc Med 2011;165:443-450.

[8] Fetus and Newborn Committee, Canadian Paediatric Society. Guidelines for transfusion of erythrocytes to neonates and premature infants. CMAJ 1992;147:17811792.

[9] Mann H. Controversial choice of a control intervention in a trial of ventilator therapy in ARDS: standard of care arguments in a randomised controlled trial. J Med Ethics 2005;31:548-553.

[10] Bayley N. Bayley scales of infant and toddler development. 3rd ed. San Antonio, TX: Harcourt Assessments, 2006.

[11] Palisano RJ, Cameron D, Rosenbaum PL, Walter SD, Russell D. Stability of the Gross Motor Function Classification System. Dev Med Child Neurol 2006;48:424-428.

[12] Achenbach TM, Rescorla LA. Manual for the ASEBA preschool forms \& profiles. Burlington: University of Vermont, Research Center for Children, Youth, \& Families, 2001.

[13] Papile LA, Burstein J, Burstein R, Koffler H. Incidence and evolution of subependymal and intraventricular hemorrhage: a study of infants with birth weights less than 1,500 gm. J Pediatr 1978;92:529-534.

[14] International Committee for the Classification of Retinopathy of Prematurity. The International Classification of Retinopathy of Prematurity revisited. Arch Ophthalmol 2005;123:991-999.

[15] Walsh MC, Wilson-Costello D, Zadell A, Newman N, Fanaroff A. Safety, reliability, and validity of a physiologic definition of bronchopulmonary dysplasia. J Perinatol 2003;23:451-456.

[16] Bell MJ, Ternberg JL, Feigin RD, et al. Neonatal necrotizing enterocolitis: therapeutic decisions based upon clinical staging. Ann Surg 1978;187:1-7.
[17] Olsen IE, Groveman SA, Lawson ML, Clark RH, Zemel BS. New intrauterine growth curves based on United States data. Pediatrics 2010;125:e214-e224.

[18] Juul SE, Comstock BA, Wadhawan R, et al. A randomized trial of erythropoietin for neuroprotection in preterm infants. N Engl J Med 2020;382:233-243.

[19] Franz AR, Engel C, Bassler D, et al. Effects of liberal vs restrictive transfusion thresholds on survival and neurocognitive outcomes in extremely low-birth-weight infants: the ETTNO randomized clinical trial. JAMA 2020;324:560-570.

[20] Andersen CC, Hodyl NA, Kirpalani HM, Stark MJ. A theoretical and practical approach to defining "adequate oxygenation" in the preterm newborn. Pediatrics 2017;139(4):e20161117-e20161117.

[21] Keir AK, Yang J, Harrison A, Pelausa E, Shah PS. Temporal changes in blood product usage in preterm neonates born at less than 30 weeks' gestation in Canada. Transfusion 2015;55:1340-1346.

[22] Maier RF, Sonntag J, Walka MM, Liu G, Metze BC, Obladen M. Changing practices of red blood cell transfusions in infants with birth weights less than 1000 g. J Pediatr 2000;136:220-224.

[23] Widness JA, Seward VJ, Kromer IJ, Burmeister LF, Bell EF, Strauss RG. Changing patterns of red blood cell transfusion in very low birth weight infants. J Pediatr 1996;129:680-687.

[24] Nunes dos Santos AM, Guinsburg R, de Almeida MF, et al. Red blood cell transfusions are independently associated with intra-hospital mortality in very low birth weight preterm infants. J Pediatr 2011;159(3):371376.e1.

[25] Kirpalani H, Whyte R. Truths, associations, and hypotheses. J Pediatr 2011;159:359-361.

[26] McGrady GA, Rettig PJ, Istre GR, Jason JM, Holman RC, Evatt BL. An outbreak of necrotizing enterocolitis: association with transfusions of packed red blood cells. Am J Epidemiol 1987;126:1165-1172.

[27] Kirpalani H, Zupancic JA. Do transfusions cause necrotizing enterocolitis? The complementary role of randomized trials and observational studies. Semin Perinatol 2012;36:269-276.

[28] Mohamed A, Shah PS. Transfusion associated necrotizing enterocolitis: a meta-analysis of observational data. Pediatrics 2012;129:529-540.

[29] Hay S, Zupancic JA, Flannery DD, Kirpalani H, Dukhovny D. Should we believe in transfusionassociated enterocolitis? Applying a GRADE to the literature. Semin Perinatol 2017;41:80-91.

[30] Patel RM, Knezevic A, Shenvi N, et al. Association of red blood cell transfusion, anemia, and necrotizing enterocolitis in very low-birth-weight infants. JAMA 2016;315:889-897.

[31] Slidsborg C, Jensen A, Forman JL, et al. Neonatal risk factors for treatment-demanding retinopathy of prematurity: a Danish national study. Ophthalmology 2016;123:796-803.

[32] Cooke RW, Drury JA, Yoxall CW, James C. Blood transfusion and chronic lung disease in preterm infants. Eur J Pediatr 1997;156:47-50.

[33] Christensen RD, Baer VL, Lambert DK, Ilstrup SJ, Eggert LD, Henry E. Association, among very-lowbirthweight neonates, between red blood cell transfusions 
in the week after birth and severe intraventricular hemorrhage. Transfusion 2014;54:104-108.

[34] Zagol K, Lake DE, Vergales B, et al. Anemia, apnea of prematurity, and blood transfusions. J Pediatr 2012;161(3):417-421.e1.

[35] Abu Jawdeh EG, Martin RJ, Dick TE, Walsh MC, Di Fiore JM. The effect of red blood cell transfusion on intermittent hypoxemia in ELBW infants. J Perinatol 2014;34:921-925.

[36] Collins R, Bowman L, Landray M, Peto R. The magic of randomization versus the myth of real-world evidence. $\mathrm{N}$ Engl J Med 2020;382:674-678.

[37] Silverman WA. Retrolental fibroplasia: a modern parable. New York: Grune \& Stratton, 1980.
[38] Fergusson D, Hébert PC, Lee SK, et al. Clinical outcomes following institution of universal leukoreduction of blood transfusions for premature infants. JAMA 2003;289:1950-1956.

[39] Fergusson DA, Hébert P, Hogan DL, et al. Effect of fresh red blood cell transfusions on clinical outcomes in premature, very low-birth-weight infants: the ARIPI randomized trial. JAMA 2012;308:1443-1451.

[40] Spinella PC, Tucci M, Fergusson DA, et al. Effect of fresh vs standard-issue red blood cell transfusions on multiple organ dysfunction syndrome in critically ill pediatric patients: a randomized clinical trial. JAMA 2019;322:2179-2190. 